\title{
EQUAÇÕES DE REGRESSÃO PARA ESTIMAÇÃO DE ALGUNS ATRIBUTOS DE SOLOS DO ESTADO DE SÃO PAULO
}

\author{
Adriana Aparecida Ribon Pinotti ${ }^{1}$ \\ José Frederico Centurion² \\ Gener Tadeu Pereira ${ }^{3}$
}

\section{RESUMO}

Foram selecionados modelos de regressão de atributos físicos, químicos e mineralógicos de amostras de cinco perfis de alguns solos do Estado de São Paulo (Latossolo Roxo, Latossolo Vermelho-Amarelo, Podzólico Vermelho-Amarelo, Areia Quartzosa e Terra Roxa Estruturada), coletadas nos municípios de Monte Alto, Jaboticabal e São Carlos. Através do método de regressão STEPWISE foram idealizadas três situações, sendo que na situação 1 , do conjunto de dez variáveis colocadas inicialmente a macroporosidade foi a que melhor explicou a relação entre a capacidade de campo e os parâmetros físicos e mineralógicos dos solos, com um $\mathrm{R}^{2}=0,8157$. Na situação 2 , o modelo utilizado para representar a relação entre $\mathrm{V} \%$ e os parâmetros químicos e mineralógicos foi explicado pelas variáveis cálcio e CTC da fração argila por Bennema dentre as nove variáveis participantes da análise, com $u m R^{2}=0,9703$. A relação entre o teor de ferro no solo e as propriedades físicas, químicas e mineralógicas na situação 3 foi explicada por um modelo composto por

1 Pós-Graduanda em Ciência do Solo da FCAV-UNESP. Câmpus de Jaboticabal, SP, Brasil.

2 Prof. Adjunto do Departamento de Solos e Adubos da FCAV-UNESP. Câmpus de Jaboticabal.

3 Prof. Assistente Doutor do Departamento de Ciências Exatas da FCAV-UNESP, Jaboticabal. 
três variáveis (hematita, areia grossa e areia fina) das nove variáveis listadas, apresentando $\mathrm{um} \mathrm{R}^{2}=0,9882$.

Palavras-chave: equações de regressão, atributos químicos, físicos, mineralógicos.

\section{ABSTRACT}

\section{REGRESSION EQUATIONS FOR THE ESTIMATION OF SOME ATTRIBUTES OF SOILS OF THE STATE OF SÃO PAULO}

Regression equations of physical, chemical and mineralogical attributes were estimated from samples of five profiles of some soils in the State of São Paulo, Brazil: Dusky Red Latosol (ACRUSTOX), Yellow Red Latosol (ACRUSTOX), Yellow Red Podzolic (KANHAPLUDALF), Quartz Sand (ULTIPSAMMENT), "Terra Roxa Estruturada" (RHODUSTALF). The samples were collected in the counties of Monte Alto, Jaboticabal and São Carlos. Through the STEPWISE regression method, three situations were idealized. In situation one, from the group of ten variables initially taken, only one variable (macroporosity) was better adjusted to the model to explain the relationship between the field capacity and the physical and mineralogical parameters of the soils, with $\mathrm{R}^{2}=0.8157$. In situation 2 , the model used to represent the relations between of $\mathrm{V} \%$ and chemical and mineralogical parameters, was explained by two variables (calcium and CEC of the clay fraction by Bennema) out of nine variables from the analysis, with a $\mathrm{R}^{2}=0.9703$. Relation between the iron content in the soil and the physical, chemical and mineralogical properties, in situation three, was explained by a model composed by three variables (hematite, coarse sand and fine sand), out of nine variables listed, presenting a $\mathrm{R}^{2}=0.9882$.

Key words: regression equations, attributes physical, chemical and mineralogical. 


\section{INTRODUÇÃO}

As propriedades físicas dos solos variam de acordo com seus constituintes minerais, principalmente no caso da mineralogia da fração argila. A caracterização e quantificação destes minerais ajudam na avaliação da gênese do solo e nas propriedades relacionadas com sua classificação e práticas agronômicas.

A classificação dos solos ou taxonomia pedológica é imprescindível para a organização científica das descobertas advindas tanto da pesquisa agronômica como da prática dos agricultores. Uma das suas vantagens é que permite, dentro de certos limites, prever o comportamento de certas terras quando usadas para determinadas finalidades. Entretanto, a caracterização dos solos, para fins de classificação e manejo, exige uma série de determinações analíticas que, muitas vezes, apresentam alto custo, além de serem demoradas. Diversas são as propriedades químicas, físicas e mineralógicas dos solos que se correlacionam entre si. $\mathrm{O}$ estudo desses atributos tem importância, notadamente dos que são dependentes (argila e água disponível; pH e saturação por bases; etc.).

Catani \& Gallo (1955) ressaltam a importância da relação entre os parâmetros químicos $\mathrm{pH}$ e V\% na determinação de métodos para a avaliação da quantidade de calcário a ser adicionada ao solo, para elevar o $\mathrm{pH}$ a um valor determinado.

Segundo Raij (1981) é surpreendente o alto grau de correlação entre a saturação por bases do solo e o pH, considerando a capacidade de troca de cátions (CTC) do solo como um reservatório ligado à escala de $\mathrm{pH}$, a qual indica o nível já atingido de bases do solo.

Silva et al. (1994), verificaram coeficientes de $\mathrm{R}^{2}=0,80^{* *}$ e $\mathrm{R}^{2}=$ $0,81^{* *}$, respectivamente, para a relação CTC - matéria orgânica, CTC conteúdo de argila do solo, em Areia Quartzosa, ao estudar perda de matéria orgânica e suas relações com a capacidade de troca catiônica, em solos cultivados com soja por 5 anos, na região de cerrados do Oeste Baiano. 
Ferreira (1988), estudando a influência da mineralogia da fração argila nas propriedades físicas de latossolos, verificou ser a macroporosidade o fator determinante da taxa de condutividade hidráulica do solo, com coeficiente de determinação altamente significativo $\left(R^{2}=0,92\right)$.

Pimentel-Gomes \& Zen (1995) estudaram a produtividade do eucalipto em função de variáveis físicas e químicas do solo através das variáveis canônicas da Análise Multivariada e do programa STEPWISE do SAS, com opção MAXR. Consideraram um total de 84 variáveis do solo (independentes) além da variável dependente representada pelo volume de madeira. Essas variáveis foram reduzidas a 25, sendo 12 da camada de 0 a $30 \mathrm{~cm}$ e 13 da camada de 30 a $60 \mathrm{~cm}$, com valores de $\mathrm{R}^{2} \mathrm{de}$ $95,70 \%$ e $89,6 \%$, respectivamente.

Outras relações, menos evidentes, mas muito úteis, devem ser conhecidas para posterior uso nas diversas especialidades da Ciência do Solo.

Assim, o trabalho teve por objetivo correlacionar os atributos nos horizontes $\mathrm{A}$ e $\mathrm{B}$ ou $\mathrm{C}$ de alguns solos do Estado de São Paulo quanto às características químicas, físicas e mineralógicas.

\section{MATERIAL E MÉTODOS}

Foram selecionadas e analisadas duas amostras de cada horizonte $\mathrm{A}$ e B ou C referentes a cinco perfis de solos do Estado de São Paulo, no total de 10 amostras, coletadas nos municípios de Monte Alto, Jaboticabal e São Carlos. As amostragens de solos e descrições morfológicas dos perfis foram realizadas até cerca de $2,0 \mathrm{~m}$, de acordo com o manual de descrição e coleta de solo no campo (Lemos \& Santos, 1996). As descrições foram feitas em unidades taxonômicas previamente definidas pela Comissão de Solos (1960), ou seja: Latossolo Roxo, Latossolo Vermelho-Amarelo, Podzólico Vermelho-Amarelo, Regosol (atual Areia Quartzosa) e Terra Roxa Estruturada.

Os métodos analíticos referentes às análises físicas e químicas seguiram as metodologias adotadas por Camargo et al. (1986). 
A análise referente ao ataque por ácido sulfúrico foi feita após digestão com $\mathrm{H}_{2} \mathrm{SO}_{4}$ 1:1, de acordo com a Embrapa (1979).

A quantificação da fração argila foi realizada de acordo com o programa computacional apresentado por Moura Filho et al. (1995).

A CTC (T) da fração argila foi determinada pelo método gráfico de Bennema (1966) e também de acordo com Camargo et al. (1987).

Foram estimadas em amostras indeformadas a retenção de umidade à tensão de $0,01 \mathrm{Mpa}$ (capacidade de campo), pelo método da centrífuga, segundo metodologia proposta por Centurion et al. (1997). Os valores referentes à tensão $1,5 \mathrm{MPa}$ (ponto de murcha permanente) foram obtidos a partir da equação de Van Genutchen, mediante programa computacional elaborado por Dourado Neto et al. (1990).

Foram realizadas três análises em três situações distintas, a saber:

Situação 1: dez variáveis independentes $=\operatorname{argila}\left(\mathrm{X}_{1}\right)$, silte $\left(\mathrm{X}_{2}\right)$, densidade do solo $\left(\mathrm{X}_{3}\right)$, densidade real $\left(\mathrm{X}_{4}\right)$, silte/argila $\left(\mathrm{X}_{5}\right)$, grau de floculação $\left(\mathrm{X}_{6}\right)$, macroporosidade $\left(\mathrm{X}_{7}\right)$, microporosidade $\left(\mathrm{X}_{8}\right)$, porosidade total $\left(\mathrm{X}_{9}\right)$ e matéria orgânica $\left(\mathrm{X}_{10}\right)$, e a variável dependente caracterizada pela retenção de água à $0,01 \mathrm{MPa}$ (capacidade de campo) representada por Y. Para esta análise, procurou-se obter um modelo com as propriedades físicas do solo que melhor explique sua relação com a capacidade de campo, propriedade fisico-hídrica importante para o uso e manejo do solo e irrigação.

Situação 2: nove variáveis independentes $=\mathrm{pH}$ em água $\left(X_{1}\right), \mathrm{pH}$ em $\mathrm{KCl}\left(\mathrm{X}_{2}\right), \Delta \mathrm{pH}\left(\mathrm{X}_{3}\right), \mathrm{Ca}^{+2}\left(\mathrm{X}_{4}\right), \mathrm{T}$ do solo $\left(\mathrm{X}_{5}\right)$, T da fração argila $\left(\mathrm{X}_{6}\right)$, CTC Bennema $\left(X_{7}\right)$, matéria orgânica $\left(X_{8}\right)$, argila $\left(X_{9}\right)$, e a variável dependente caracterizada pela saturação por bases (V\%) representada por Y. A relação das variáveis independentes com a propriedade química $V \%$ é importante, uma vez que esta propriedade é utilizada no Sistema de Classificação de Solos par a sua divisão em classes, caracterizadas quanto à fertilidade (distróficos ou eutróficos).

Situação 3: nove variáveis independentes $=\operatorname{argila}\left(\mathrm{X}_{1}\right)$, silte $\left(\mathrm{X}_{2}\right)$, 
$\mathrm{V} \%\left(\mathrm{X}_{3}\right)$, matéria orgânica $\left(\mathrm{X}_{4}\right)$, areia grossa $\left(\mathrm{X}_{5}\right)$, areia fina $\left(\mathrm{X}_{6}\right), \mathrm{Ki}\left(\mathrm{X}_{7}\right)$, goetita $\left(\mathrm{X}_{8}\right)$, hematita $\left(\mathrm{X}_{9}\right)$, e o conteúdo de ferro total $(\mathrm{Y})$, considerado como variável dependente. $\mathrm{O}$ estudo da relação destas variáveis com 0 teor de ferro no solo é importante, pois esta variável, considerada no Sistema de Classificação de Solos, é um parâmetro muito utilizado para os solos tropicais, principalmente para Latossolos.

A análise de regressão STEPWISE foi usada para modelar os efeitos das propriedades do solo nas três situações. Para tanto, aplicou-se o procedimento PROC REG, com opção MAXR do Sistema SAS (1990).

Utilizou-se a simbologia ns, *,** nos modelos obtidos, para representar, respectivamente: não significativo, significativo ao nível de 5\% de probabilidade e significativo ao nível de $1 \%$ de probabilidade, para indicar a significância dos coeficientes das equações de regressão.

\section{RESULTADOS E DISCUSSÃO}

Através do coeficiente de determinação $\left(\mathrm{R}^{2}\right)$, o qual mede a proporção da Soma de Quadrados da variável dependente (Y), explicada pelo modelo de regressão (Draper \& Smith, 1981), selecionaram-se as variáveis para as situações 1,2 , e 3 .

$\mathrm{Na}$ situação 1, a equação obtida para estimativa da retenção de água a $0,01 \mathrm{MPa}(\mathrm{Y})$ incluiu apenas a variável macroporosidade $\left(\mathrm{X}_{7}\right)$, com $\mathrm{R}^{2}=0,8157$.

O modelo estimado foi dado por:

$$
\mathrm{Y}=-2,54^{\mathrm{ns}}+1,22^{* *} \mathrm{X}_{7}
$$

O potencial de 0,01 MPa é considerado o ponto de capacidade de campo, no qual a água retirou-se dos poros maiores ou macroporos, dando espaço para que sejam preenchidos por ar (Brady, 1973). 
Estes resultados indicaram que a alteração na estrutura afeta a capilaridade e a distribuição de tamanho dos poros do solo, conforme relatam Scardua (1972) e Jaccoud \& Castro (1976). Esses autores verificaram que a estrutura desempenha papel muito importante na capacidade de retenção de água, atuando na retenção a baixa tensão, devido à presença de macroporos em poros entre agregados. A Tabela 1 apresenta os valores de retenção de água a $0,01 \mathrm{MPa}$ e da macroporosidade.

Tabela 1. Valores das variáveis selecionadas pelo método STEPWISE, com opção MAXR, na situação 1, expressas em porcentagem Volumétrica.

\begin{tabular}{|c|c|c|c|}
\hline Solos & Horizonte & Retenção de água a 0,001 MPa & Macroporosidade \\
\hline \multirow[t]{2}{*}{ Areia Quartzosa } & Ap & 15,3 & 18,8 \\
\hline & $\mathrm{C}_{2}$ & 17,8 & 22,2 \\
\hline \multirow[t]{2}{*}{ Latossolo Roxo } & $A p_{1}$ & 39,5 & 34,0 \\
\hline & $\mathrm{Bw}_{2}$ & 41,5 & 34,3 \\
\hline \multirow[t]{2}{*}{ Latossolo Vermelho-Amarelo } & $A p$ & 31,1 & 22,0 \\
\hline & $\mathrm{Bw}_{2}$ & 32,8 & 28,8 \\
\hline \multirow[t]{2}{*}{ Terra Roxa Estruturada } & Ap & 50,0 & 43,4 \\
\hline & $\mathrm{Bt}_{1}$ & 45,8 & 45,8 \\
\hline \multirow[t]{2}{*}{ Podzólico Vermelho-Amarelo } & Ap & 46,1 & 37,3 \\
\hline & $\mathrm{Bt}_{\mathrm{l}}$ & 48,1 & 35,0 \\
\hline
\end{tabular}

Na situação 2, a primeira variável que melhor estima a variação de $V \%$ é cálcio $\left(X_{4}\right), c o m R^{2}=0,9305$. Isto evidencia a forte influência deste elemento na variável dependente, pois o cálcio é o cátion trocável que melhor representa a saturação por bases dos solos tropicais em função do material de origem dos solos estudados (basalto e arenito). Com a seleção da variável CTC da fração argila obtida pelo método de Bennema $\left(\mathrm{X}_{7}\right)$ há um incremento significativo no $\mathrm{R}^{2}$ para 0,9703 . O método gráfico de Bennema (1966), leva em consideração, para a obtenção da T da fração argila, o conteúdo de argila e carbono dos solos. Isto pode explicar o incremento na estimativa do coeficiente de determinação com a entrada desta variável, pois como o conteúdo de carbono é obtido através do teor de matéria orgânica do solo, esta, através da sua mineralização, libera 
cálcio, evidenciando a relação entre a CTC da fração argila por Bennema e o conteúdo de cálcio, que, como já foi frisado, é a principal base que contribui para a elevação da saturação por bases.

O modelo estimado para esta situação foi:

$Y=16,19 * *+0,78 * * X_{4}-1,13 * X_{7}$

A Tabela 2 apresenta as variáveis utilizadas no modelo da situação 2.

Tabela 2. Valores das variáveis selecionadas pelo método STEPWISE, com opção MAXR, na situação 2.

\begin{tabular}{|c|c|c|c|c|}
\hline \multirow[t]{2}{*}{ Solos } & \multirow[t]{2}{*}{ Horizonte } & $\begin{array}{l}\text { Saturação } \\
\text { Por Bases }\end{array}$ & $\mathrm{Ca}^{2+}$ & $\begin{array}{c}\text { CTC } \\
\text { Bennema }\end{array}$ \\
\hline & & $\%$ & $\mathrm{~m} \cdot \mathrm{mol}_{\mathrm{c}} \mathrm{dm} \mathrm{m}^{-3}$ & g. $100 \mathrm{~g}^{-1}$ \\
\hline \multirow[t]{2}{*}{ Areia Quartzosa } & Ap & 11 & 2 & 4,8 \\
\hline & $\mathrm{C}_{2}$ & 10 & 2 & 4,8 \\
\hline \multirow[t]{2}{*}{ Latossolo Roxo } & $\mathrm{Ap}_{1}$ & 32 & 17 & $-1,2$ \\
\hline & $\mathrm{Bw}_{2}$ & 31 & 4 & $-1,2$ \\
\hline \multirow[t]{2}{*}{ Latossolo Vermelho-Amarelo } & Ap & 12 & 4 & 2,9 \\
\hline & $\mathrm{Bw}_{2}$ & 11 & 2 & 2,9 \\
\hline \multirow[t]{2}{*}{ Terra Roxa Estruturada } & Ap & 69 & 85 & 10,0 \\
\hline & $\mathrm{Bt}_{1}$ & 72 & 78 & 10,0 \\
\hline \multirow[t]{2}{*}{ Podzólico Vermelho-Amarelo } & Ap & 62 & 53 & $-3,6$ \\
\hline & $\mathrm{Bt}_{1}$ & 73 & 73 & $-3,6$ \\
\hline
\end{tabular}

Na situação 3, a primeira variável selecionada que mais explicou o conteúdo de ferro total $(Y)$ foi a hematita $\left(X_{5}\right)$ com $R^{2}=0,9279$, evidenciando relação direta entre esta variável e o conteúdo de ferro total (Y). A segunda variável selecionada foi a areia fina $\left(\mathrm{X}_{6}\right)$, aumentando a estimativa de $\mathrm{R}^{2}$ para 0,9575 . Finalmente a terceira variável selecionada foi a areia grossa $\left(X_{7}\right) \operatorname{com} R^{2}=0,9882$. Isto pode ser explicado provavelmente pela relação inversa e significativa entre o teor de $\mathrm{Fe}_{2} \mathrm{O}_{3}$ e o conteúdo de areia grossa. De acordo com Oliveira \& Menk (1984), o fato de a areia 
grossa ser composta essencialmente de quartzo, provoca diluição dos minerais fornecedores de ferro, acarretando, em consequência, teores menos elevados de ferro total.

O modelo estimado com estas três variáveis foi dado por:

$$
\mathrm{Y}=201,5017 *-0,2150 * * \mathrm{X}_{5}-0,2358 * * \mathrm{X}_{6}+0,5345 * * \mathrm{X}_{9}
$$

A Tabela 3 apresenta as variáveis utilizadas na situação 3.

Tabela 3. Valores das variáveis selecionadas pelo método STEPWISE, com opção MAXR, na situação 3.

\begin{tabular}{|c|c|c|c|c|c|}
\hline \multirow[t]{2}{*}{ Solos } & \multirow[t]{2}{*}{ Horizonte } & $\mathrm{Fe}_{2} \mathrm{O}_{3}$ & Hematita & Areia Grossa & Areia Fina \\
\hline & & \multicolumn{4}{|c|}{$\mathrm{g} . \mathrm{kg}^{-1}$} \\
\hline \multirow[t]{2}{*}{ Areia Quartzosa } & $\mathrm{Ap}$ & 8 & 0 & 210 & 680 \\
\hline & $\mathrm{C}_{2}$ & 8 & 0 & 240 & 660 \\
\hline \multirow[t]{2}{*}{ Latossolo Roxo } & $A p_{1}$ & 311 & 219 & 20 & 90 \\
\hline & $\mathrm{Bw}_{2}$ & 329 & 311 & 10 & 70 \\
\hline \multirow[t]{2}{*}{ Latossolo Vermelho-Amarelo } & $A p$ & 51 & 0 & 360 & 310 \\
\hline & $\mathrm{Bw}_{2}$ & 69 & 0 & 310 & 250 \\
\hline \multirow[t]{2}{*}{ Terra Roxa Estruturada } & $\mathrm{Ap}$ & 255 & 177 & 40 & 150 \\
\hline & $\mathrm{Bt}_{\mathrm{l}}$ & 255 & 136 & 30 & 110 \\
\hline \multirow[t]{2}{*}{ Podzólico Vermelho-Amarelo } & Ap & 19 & 0 & 140 & 610 \\
\hline & $\mathrm{Bt}_{1}$ & 29 & 0 & 130 & 510 \\
\hline
\end{tabular}

Assim, pela situação 1, através da determinação da macroporosidade é possível, com elevado grau de precisão, estimar a retenção de água do solo a $0,01 \mathrm{MPa}$. Considerando a facilidade e rapidez na determinação da macroporosidade, a aplicação do modelo torna-se viável.

A situação 2 evidencia a participação da variável CTC da fração argila, obtida pelo método gráfico de Bennema (1966) no modelo mencionado. De acordo com Prado (1993), o método gráfico de Bennema é mais indicado para solos que possuam horizonte $\mathrm{Bw}$, isto é, solos em estágios bem avançados de intemperização. Para a obtenção dos modelos 
com maiores valores de coeficiente de determinação, houve a contribuição de parâmetros provindos das amostras de solos com diferentes estágios de evolução, desde solos mais jovens, como os litólicos, até solos ácricos, isto é, extremamente intemperizados. Certamente, se somente os dados das amostras dos solos com um elevado grau de intemperização (Latossolo Roxo, Latossolo Vermelho-Amarelo) tivessem participado das análises, maior coeficiente de determinação seria obtido para a relação entre CTC da fração argila e as outras variáveis do modelo.

De acordo com a situação 3 , torna-se possível estimar o conteúdo de ferro total $\left(\mathrm{Fe}_{2} \mathrm{O}_{3}\right)$ dos solos, devido a relação inversa entre $\mathrm{Fe}_{2} \mathrm{O}_{3}$ e o conteúdo de areia grossa. Deste modo, pelo modelo proposto pela análise 3 , solos com textura mais argilosa apresentam teores mais elevados de ferro total. Com a determinação da composição granulométrica (análise textural), relativamente simples e de rotina nos laboratórios de análises de solos, consegue-se, pelo modelo proposto, a estimativa do conteúdo de $\mathrm{Fe}_{2} \mathrm{O}_{3}$.

\section{CONCLUSÕES}

Para a situação 1 , do conjunto de dez variáveis colocadas inicialmente, a variável macroporosidade $\left(\mathrm{X}_{7}\right)$ foi a que melhor se ajustou ao modelo para explicar a relação entre a capacidade de campo e os parâmetros físicos e mineralógicos dos solos.

Na situação 2, modelo de regressão estimado para representar a relação entre $\mathrm{V} \%$ e os parâmetros químicos e mineralógicos foi composto pelas variáveis cálcio $\left(\mathrm{X}_{4}\right)$ e CTC da fração argila por Bennema $\left(\mathrm{X}_{7}\right)$ dentre as nove variáveis participantes da análise.

A relação entre o teor de ferro no solo e as propriedades físicas, químicas e mineralógicas, na situação 3 , foi explicada por um modelo composto por três variáveis: hematita $\left(\mathrm{X}_{9}\right)$, areia grossa $\left(\mathrm{X}_{5}\right)$ e areia fina $\left(\mathrm{X}_{6}\right)$, das nove variáveis relacionadas.

As equações estimadas que melhor representaram as variáveis dependentes (capacidade de campo, saturação por bases e teor de ferro) 
para as situações 1, 2 e 3, respectivamente, foram:

$$
\begin{aligned}
& Y=-2,54^{n s}+1,22^{* *} X_{7},\left(R^{2}=0,8157\right) ; \\
& Y=16,19^{* *}+0,78^{* *} X_{4}-1,13^{*} X_{7},\left(R^{2}=0,9703\right) ; \\
& Y=201,5017^{* *}-0,2150^{* *} X_{5}-0,2358^{* *} X_{6}+0,5345^{* *} X_{9},\left(R^{2}=0,9882\right) .
\end{aligned}
$$

\section{REFERÊNCIAS BIBLIOGRÁFICAS}

BENNEMA, J., 1966. The Calculation of CEC for 100 Grams of Clays With Correction for Organic Carbon. In: CLASSIFICATION OF BRAZILIAIN SOILS. Roma: FAD. p.27-30. (EPTA Report 2127).

BRADY, N.C., 1983. Natureza e Propriedade dos Solos. 6 ed., São Paulo, Biblioteca Universitária Freitas Bastos. 647p.

CAMARGO, O.A.; A.C. MONIZ; J.A. JORGE; J.M.A.S. VALADARES, 1986. Métodos de Análise Química, Mineralógica e Física de Solos do Instituto Agronômico de Campinas. Campinas, IAC, 94p. (Boletim Técnico, 106).

CAMARGO, M.N. ; E. KLAMT; J.H. KAMFFMAN, 1987. Classificação de Solos Usados em Levantamentos Pedológicos no Brasil. Separata do Bol. Inf. Soc. Bras. Cienc. Solo, Campinas, 12 (1):11-33.

CATANI, R.A.; J.R. GALLO, 1955. A Avaliação da Exigência em Calcário dos Solos do Estado de São Paulo Mediante Correlação entre pH e Porcentagem de Saturação por Bases. Rev. Agric.; Piracicaba, 30: 49-60.

CENTURION, J.F.; M.H. MORAES; C.L.F. DELLA LIBERA,.1997.

Comparação de Métodos para Determinação da Curva de Retenção de Água em Solos. Rev. Bras. Ciênc. Solo, Campinas, 21: 173-179. DOURADO NETO, D.; Q. JONG VAN LIER; T.A. BOTREL; P.L. LIBARDI, 1990. Programa para Confecção de Curva de Retenção de Água, Utilizando Modelo de Genuchten. Eng. Rural, Piracicaba, 1(2): 92-102. DRAPER, N.R.; H. SMITH, 1981. Applied Regression Analysis. New York, John Wiley. 709p. 2 edition. 
EMBRAPA, 1979. Serviço Nacional de Levantamento e Conservação do

Solo. Manual de Métodos de Análises de Solo. Rio de Janeiro, s.p. FERREIRA, M. .M., 1988. Influência da Mineralogia da Fração Argila nas Propriedades Físicas de Latossolos Brasileiros. Viçosa. p.79. Tese (Doutorado-UFV).

JACCOUD, A.; F. CASTRO, 1976. Curvas de Caracterização de Umidade de Solos da Área da Universidade Federal Rural do Rio de Janeiro, Município de Itajai. Pesq. Agropec. Bras., Brasília, 11:. 1-9.

LEMOS, R.C.; R.D. SANTOS, 1996. Manual de Descrição e Coleta de Solo no Campo. 3.ed. Campinas: Sociedade Brasileira de Ciência do Solo, SNLCS. 84 p.

MOURA FILHO, G.; M. RESENDE, C.D.CRUZ, 1995. Aloca. Programa para Estimativa de Minerais do Solo. In: CONGRESSO BRASILEIRO DE CIÊNCIA DO SOLO, 25, Viçosa. Anais...

OLIVEIRA, J.B.; J.R.F. MENK., 1984. Latossolos Roxos do Estado de São Paulo. Campinas, Instituto Agronômico. 135p. (Boletim Técnico, 82). PIMENTEL-GOMES, F.; S. ZEN., 1995. A Produtividade de Eucalipto em Função de Variáveis Físicas e Químicas do Solo. In: XXV CONGRESSO BRASILEIRO DE CIÊNCIA DO SOLO, 2241, Viçosa. Anais... PRADO, H., 1993. Manual de Classificação de Solos do Brasil. Jaboticabal, FUNEP p.218.

RAIJ, B. van., 1981. Avaliação da Fertilidade do Solo. Piracicaba: Instituto da Potassa e do Fosfato, Instituto Internacional da Potassa, 142p. SAS INSTITUTE INC., 1990. SAS/STAT Users Guide. Version 6. SAS Inst. Cary, N C.

SCARDUA, R., 1972. Porosidade de Água em Dois Solos do Município de Piracicaba - SP. Piracicaba, 83p. Dissertação (Mestrado em Solos e Nutrição de Plantas) - Esalq/USP.

SILVA, J.E.; J. LEMAINSKI,.; D.V.S. RESCK, 1994. Perdas de Matéria Orgânica e Suas Relações com a CTC em Solos da Região de Cerrados do Oeste Baiano. Rev. Bras. Ciência Solo, Campinas, 18: 541-547. 